\title{
An improved model to study tumor cell autonomous metastasis programs using MTLn3 cells and the Rag2 ${ }^{-1-} \mathrm{\gamma c}^{-/-}$mouse
}

\author{
Sylvia E. Le Dévédec · Wies van Roosmalen · \\ Naomi Maria · Max Grimbergen - Chantal Pont • \\ Reshma Lalai · Bob van de Water
}

Received: 14 November 2008/Accepted: 24 April 2009/Published online: 24 May 2009

(C) The Author(s) 2009. This article is published with open access at Springerlink.com

\begin{abstract}
The occurrence of metastases is a critical determinant of the prognosis for breast cancer patients. Effective treatment of breast cancer metastases is hampered by a poor understanding of the mechanisms involved in the formation of these secondary tumor deposits. To study the processes of metastasis, valid in vivo tumor metastasis models are required. Here, we show that increased expression of the EGF receptor in the MTLn3 rat mammary tumor cell-line is essential for efficient lung metastasis formation in the Rag mouse model. EGFR expression resulted in delayed orthotopic tumor growth but at the same time strongly enhanced intravasation and lung metastasis. Previously, we demonstrated the critical role of NK cells in a lung metastasis model using MTLn3 cells in syngenic F344 rats. However, this model is incompatible with human EGFR. Using the highly metastatic EGFRoverexpressing MTLn3 cell-line, we report that only $\operatorname{Rag} 2^{-1-} \gamma c^{-1-}$ mice, which lack NK cells, allow efficient lung metastasis from primary tumors in the mammary gland. In contrast, in nude and SCID mice, the remaining innate immune cells reduce MTLn3 lung metastasis formation. Furthermore, we confirm this finding with the orthotopic transplantation of the 4T1 mouse mammary tumor cell-line. Thus, we have established an improved in vivo model using a $\mathrm{Rag}^{-1-} \gamma \mathrm{c}^{-/-}$mouse strain together
\end{abstract}

Electronic supplementary material The online version of this article (doi:10.1007/s10585-009-9267-6) contains supplementary material, which is available to authorized users.

S. E. Le Dévédec · W. van Roosmalen · N. Maria ·

M. Grimbergen · C. Pont · R. Lalai · B. van de Water ( $₫)$

Division of Toxicology, Leiden Amsterdam Center for Drug

Research (LACDR), Leiden University, P.O. Box 9502, 2300

RA Leiden, The Netherlands

e-mail: water_b@lacdr.leidenuniv.nl with MTLn3 cells that have increased levels of the EGF receptor, which enables us to study EGFR-dependent tumor cell autonomous mechanisms underlying lung metastasis formation. This improved model can be used for drug target validation and development of new therapeutic strategies against breast cancer metastasis formation.

Keywords Breast cancer - EGF receptor - ErbB1 .

Metastasis - MTLn3 cells $\cdot 4 \mathrm{~T} 1$ cells .

Immune deficient mice

\author{
Abbreviations \\ SCID Mice severe combined immunodeficiency mice \\ RAG2 Recombinase activating gene \\ $\gamma \mathrm{c} \quad$ Common gamma chain \\ FACS Fluorescence activated cell sorter \\ ErbB1 Epidermal growth factor receptor \\ GFP Plasmid green fluorescent protein \\ IL-2 Interleukin 2 \\ MHC Major histocompatibility complex \\ NK Cell natural killer cell \\ FLI Fluorescent imaging \\ APC Allophycocyanin
}

\section{Introduction}

Breast cancer is the most common cause of cancer among women and the second leading cause of cancer deaths in Western countries. The metastatic spread of tumor cells from their primary site to distant organs in the body is the principal cause of mortality [1]. Thus, understanding metastasis is one of the most significant problems in cancer research [2-4]. A key challenge is to develop suitable 
animal models to enhance our understanding of the mechanisms that underlie metastatic progression and to evaluate treatments for metastatic diseases [5].

Currently available in vivo models of breast tumor progression and metastasis include transplantable models and genetically engineered mice that develop primary and metastatic cancers [5-8]. Transplantable tumor models include syngeneic models, in which the cancer cell line/ transplanted tissue is of the same genetic background as the animal, and xenograft models whereby human cancer cell lines or tissues are transplanted into immunocompromised hosts, such as nude and severe combined immunodeficient mice [5]. Breast xenograft tumors are produced by injecting breast cancer cells into the flank (subcutaneous) or preferably into the mammary fat pad (orthotopic) of a female animal. Subcutaneous xenograft mouse models are typically the standard for cancer drug screening in the pharmaceutical industry [9], but the use of orthotopic xenotransplantation models should be favored since tissue specific stromal cell interactions play a crucial role in the biology of cancer progression and metastasis.

Metastasis is a consequence of multiple steps, including growth of a primary tumor, intravasation, arrest and growth in a secondary site $[2,3]$. The study of metastasis requires both a relevant mouse model and an appropriate tumor cell line. On the basis of gene profiling and previous in vivo results, rat mammary adenocarcinoma MTLn3 cells have been identified as a suitable model to study breast cancer progression and treatment $[10,11]$. The epidermal growth factor receptor (EGFR, also referred as ErbB1) is often overexpressed in breast cancer, resulting not only in uncontrolled cell proliferation $[12,13]$ but also in increased tumor cell motility and invasion [14-16]. To study intravasation leading to metastasis formation, we therefore evaluated the effect of enhanced ErbB1 signaling in MTLn3 cells in the orthotopic Rag mouse breast cancer model.

Efficient metastasis formation is dependent on tumor cell autonomous biological programs that define migration, intravasation survival and extravasation [14]. For xenotransplantation models, immune cell responses to foreign antigens on the injected tumor cells need to be avoided, necessitating the use immunocompromised hosts.

The most widely used immunodeficient mice (nude and SCID) lack the adaptive immune response. However, these mice still harbor large numbers of cells of the innate immune system, including natural killer (NK) cells [17]. These cells are important in the killing of viable circulating tumor cells that, through enhanced invasion and intravasation programs, have efficiently escape the primary tumor and have the potential to form metastasis [18-25]. Indeed, we showed previously that the MTLn3 cells are killed by the circulating NK-cells in Fischer 344 rats, thus preventing efficient lung metastasis formation. Although pretreatment with NK-depleting antibodies allowed experimental lung metastasis formation in this model [26, 27], continuous NK depleting antibody injection is an undesirable requirement for a breast/tumor metastasis model. To study breast tumor progression and metastasis formation using MTLn3 cells expressing the EGF receptor, an appropriate animal model with a compromised innate and adaptive immune system was still required.

The goal of the current study was to develop an MTLn3 cell breast tumor metastasis animal model that allows the unbiased analysis of tumor cell-dependent metastasis programs, independent of adaptive and innate immune system surveillance. We first show that increased expression of the ErbB1 receptor in MTLn3 cells was required for lung metastasis formation in Rag2 $2^{-/-} \gamma c^{-/-}$mice. Secondly, by comparing different immune deficient mouse models, we confirm that Rag2 ${ }^{-1-} \gamma \mathrm{c}^{-1-}$ mice, which lack NK cells, are an ideal recipient animal model for MTLn3 and 4T1 cells to study the metastasis process. In conclusion, we have established an improved animal model that can be used to study the biological steps that are essential in the formation of distant metastasis.

\section{Materials and methods}

\section{Cell lines}

MTLn3 rat mammary adenocarcinoma cells [28] were cultured as previously described [29]. MTLn3-GFP-ErbB1 and MTLn3-GFP cell-lines were previously described [16] and were maintained in $\alpha$ MEM (Life Technologies, Inc., Gaithersburg, MD) supplemented with 5\% fetal bovine serum (Life Technologies). The mouse mammary metastatic 4T1-luc cell-line was purchased from Caliper Lifescience and cultured in RPMI-1640 supplemented with $10 \%$ fetal bovine serum (Life Technologies).

\section{Reagents}

Mouse anti-human ErbB1 was purchased from Calbiochem (EMD Biosciences, San Diego, California). Rabbit antihuman ErbB1 used for immunoblotting was purchased from Cell Signaling Technology (Danvers, MA). Goat antimouse APC was purchased from Cedarlane (Ontario, Canada). Alpha modified minimal essential medium (aMEM), Fetal Bovine Serum (FBS), phosphate buffered saline (PBS) and trypsin were from Life Technologies (Rockville, MD, USA). 
ErbB1 staining

\section{Flow cytometry}

MTLn3 were harvested and incubated for 45 min with $50 \mu \mathrm{l}$ of $\mathrm{mAb}$ ErbB1 $(2 \mu \mathrm{g} / \mathrm{ml}$ in PBS). Cells were washed with cold PBS and incubated for 45 min with the secondary antibody goat-anti mouse APC in PBS $(2 \mu \mathrm{g} / \mathrm{ml})$. Finally, cells were washed and suspended in $0.3 \mathrm{ml}$ PBS. ErbB1 and GFP expression were analysed by flow cytometry (FACScalibur, Becton Dickinson).

\section{Immunoblotting}

Cells were scraped in ice-cold TSE (10 nM Tris-HCl, $250 \mathrm{mM}$ sucrose, $1 \mathrm{mM}$ EGTA, pH 7.4) supplemented with inhibitors. After sonication of either cells or tissue, protein concentrations were determined by the Bio-Rad (Hercules, CA) protein assay using IgG as a standard. Equal amounts of total cellular protein were separated by 7.5\% SDS-PAGE and transferred to polyvinylidene difluoride membranes (Millipore, Billerica, MA). Blots were blocked with $5 \%(\mathrm{w} / \mathrm{v})$ bovine serum albumin in TBST $[0.5 \mathrm{~mol} / \mathrm{l} \mathrm{NaCl}, 20 \mathrm{mmol} / \mathrm{l}$ Tris- $\mathrm{HCl}, 0.05 \%$ (v/v) Tween $20(\mathrm{pH} \mathrm{7.4)]} \mathrm{and} \mathrm{probed} \mathrm{with} \mathrm{primary} \mathrm{antibody}$ (overnight, $4^{\circ} \mathrm{C}$ ) followed by incubation with secondary horseradish peroxidase-coupled antibody and visualized with Enhanced Chemiluminescence Plus reagent (Amersham Biosciences, Uppsala, Sweden) by scanning on a multilabel Typhoon imager 9400 (Amersham Biosciences).

\section{Animals}

Female BALB/c nu/nu, SCID [CB17/lcr-Prkdc ${ }^{\text {scid } / C r l] ~ a n d ~}$ SCID Beige [CB17/lcr.Cg-Prkdc ${ }^{\text {scid }}$ Lyst $\left.^{\text {bg }} / \mathrm{Crl}\right]$ mice aged between 6 and 7 weeks were purchased from Charles River (L'Arbresle, France). BALB/c mice aged between 6 and 7 weeks were purchased from Janvier (Uden, The Netherlands). 6-week old Rag2 $2^{-l-} \gamma \mathrm{c}^{-/-}$mice were obtained from in house breeding. Animals were housed in individually ventilated cages under sterile conditions containing three mice per cage. Sterilised food and water were provided ad libitum.

Spontaneous and experimental metastasis assays

To measure spontaneous metastasis, tumor cells were grown to $70-85 \%$ confluence before being harvested for cell counting. Cells $\left(5 \times 10^{5}\right.$ for the MTLn 3 or $1 \times 10^{5}$ for the 4T1-luc) were injected into the right thoracic mammary fat pads of the different mouse strains. Cells were injected in a volume of $100 \mu \mathrm{l}$ of PBS without $\mathrm{Ca}^{2+}$ and $\mathrm{Mg}^{2+}$ through a 25-gauge needle. Tumor growth rate was monitored at weekly intervals after inoculation of tumor cells. Horizontal $(h)$ and vertical $(v)$ diameters were determined, and tumor volume $(V)$ was calculated $(V=4 /$ $3 \pi\left(1 / 2[\sqrt{ }(h \times v)]^{3}\right)$. After 3 or 4 weeks, the animals were anesthetized with pentobarbital and the lungs were excised and rinsed in ice-cold PBS. For the GFP-labeled MTLn3 cell-lines, the right lung was used to count the tumor burden. For rough estimation, the right lungs were imaged with the Fluorescent imaging unit IVIS (see below). And for detailed quantification, the flat side of the right lung was analysed with the immunofluorescence microscope. With a $\times 10$ objective lens, we screened the flat surface of the lobe and counted the number of GFP positive metastases. Subsequently, the right lung was fixed in $4 \%$ paraformaldehyde. The left lung was injected with ink solution, de-stained in water and fixed in Fekete's (4.3\% (v/v) acetic acid, $0.35 \%(\mathrm{v} / \mathrm{v})$ formaldehyde in $70 \%$ ethanol). For the 4T1-luc cell-line, the lung tumor burden was quantified by counting the number of surface metastases which were represented by white spots on the ink-injected left lung.

For the experimental lung metastasis assay, $2 \times 10^{5}$ MTLn3 cells were injected into the lateral tail veins of 5- to 7 -week-old female $\mathrm{Rag} 2^{-1-} \gamma \mathrm{c}^{-1-}$ mice. Three to four weeks after injection, the mice were euthanized, and the lungs were removed and subjected to fluorescent imaging and histologic examination as described below.

\section{Fluorescent imaging}

Fluorescent imaging (FLI) was performed with a high sensitivity, cooled CCD camera mounted in a light-tight specimen box (IVIS ${ }^{\mathrm{TM}}$; Xenogen). Imaging and quantification of signals were controlled by the acquisition and analysis software Living Image ${ }^{\circledR}$ (Xenogen). For ex vivo imaging, lungs were excised, placed into a petri dish, and imaged for 1-2 min. Tissues were subsequently fixed as above and prepared for standard histopathology evaluation.

\section{Measurement of tumor cell blood burden}

At the end point of the MTLn3 spontaneous metastasis assay, mice were sacrificed with Nembutal. The right side of the thoracic cavity was exposed by a simple skin flap surgery. Blood was taken from the right atrium via heart puncture with a 25 -gauge needle and $1-\mathrm{ml}$ syringe coated with heparin. Blood (0.2-1.0 ml) was harvested from each animal. The blood was immediately plated into $100-\mathrm{mm}$ diameter dishes filled with $5 \%$ fetal bovine serum/ $\alpha$ MEM growth medium. The next day, the plates were rinsed with fresh medium containing $0.8 \mathrm{mg} / \mathrm{ml}$ geneticin to selectively grow the tumor cells. After 3-7 days, all dishes were scanned for GFP expression with a Typhoon imager 9400 (Amersham Biosciences). The tumor cell clones were 
counted by image analysis of the obtained scans. Tumor blood burden was calculated as total colonies in the dish divided by the volume of blood taken.

Tumor histology and quantitative assessment of the efficiency of metastasis

The primary tumors and lungs from each mouse were used for histological analysis. Samples were fixed in formalin and embedded in paraffin, and 5- $\mu \mathrm{m}$ sections were stained with H\&E.

\section{Statistical analysis}

Student's $t$ test was used to determine if there was a significant difference between two means $(P<0.05)$. Values are presented as mean $\pm \mathrm{SD}$. Significant differences are marked in the graphs.

\section{Results}

ErbB1 overexpression in MTLn3 cells delays tumor growth but facilitates breast cancer lung metastasis formation in $\operatorname{Rag} 2^{-1-} \gamma \mathrm{c}^{-1-}$ mice

Since the EGF receptor is often over expressed in breast cancer progression [12, 13], we used MTLn3-GFP-ErbB1 cells, because of the high likelihood of success for metastasis formation. First, we verified whether ErbB1 overexpression is indeed essential for metastases formation in the Rag mouse model. Therefore we compared MTLn3GFP with MTLn3-GFP-ErbB1 cells. Increased expression of ErbB1 protein in the MTLn3-GFP-ErbB1 cell-line was confirmed by Western blot analysis (Fig. 1a) and flow cytometry (Fig 1b). We also confirmed that GFP was equally expressed in both cell-lines (Fig. 1b). The expression of GFP was used to count the lung metastases and
Fig. 1 ErbB1 over-expression affects tumor growth rate of MTLn3 in a spontaneous metastasis assay. a Western blot of ErbB1 expression in MTln3GFP and MTLn3-GFP-ErbB1 cell-lines. b Fluorescenceactivated cell sorting (FACS) analysis of cell surface expression levels of human ErbB1 and GFP expression levels for MTLn3-GFP and MTLn3-GFP-ErbB1 cell-lines. c Primary tumor growth measured by caliper during spontaneous metastasis assay. Rag mice were inoculated with MTLn3-GFP $(n=13)$ and MTLn3-GFP-ErbB1 $(n=17)$ cells (MTln3-GFP-ErbB1 vs. MTLn3-GFP, $P<0.0001)$. d Primary tumor weight at the end point of the spontaneous metastasis assay $(P>0.05)$ (a)

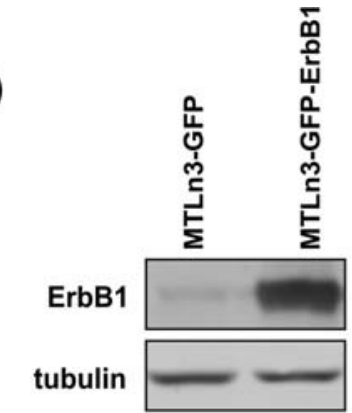

(b)
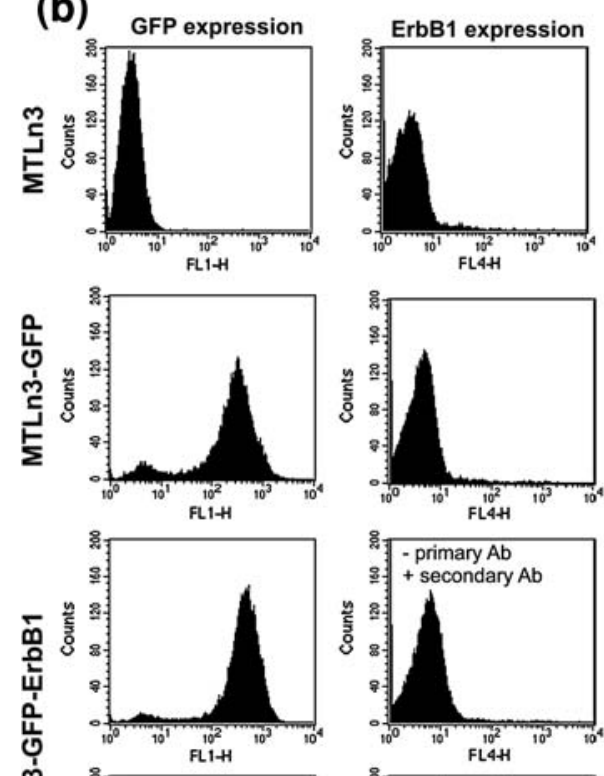

党

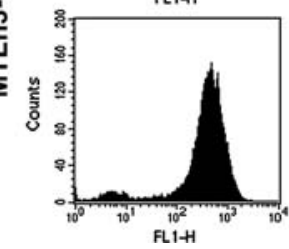

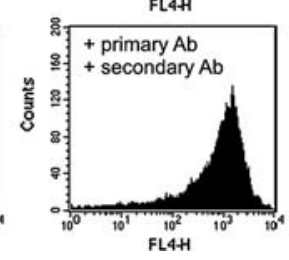

(c)

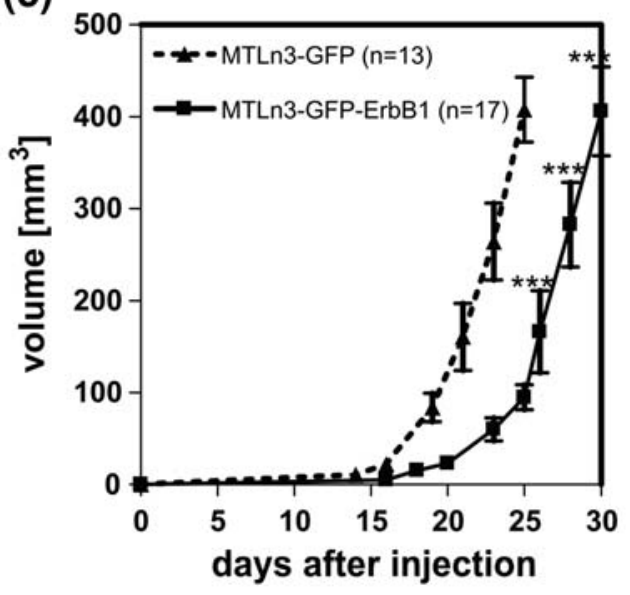

(d)

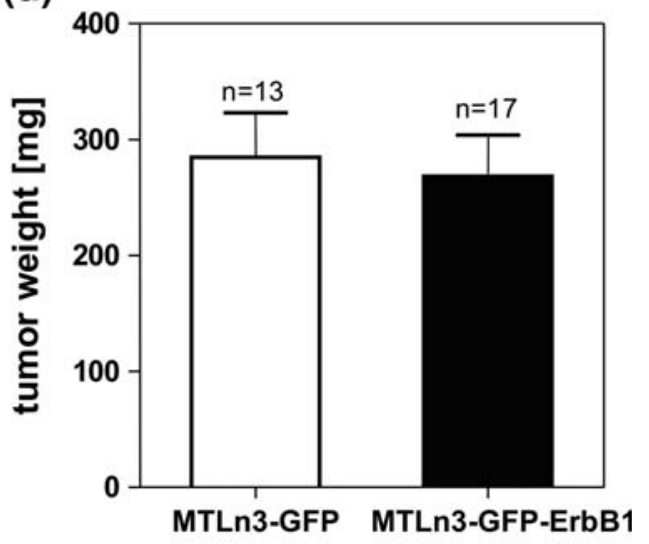


Fig. 2 ErbB1 over-expression significantly enhances lung metastasis formation

independent of tumor weight. a Lung metastases counted with a fluorescent microscope at $\times 10$ magnification (only one side of the left lung) at the end point of the spontaneous metastasis assay (MTln3-GFP-ErbB1 versus MTLn3-GFP,

$P<0.0001)$. b Lung metastases visualized with the FLI (GFP detection) after spontaneous metastasis assay with the MTLn3-GFP and the MTLn3GFP-ErbB1 cells. c H\&E staining of lung tissue of MTLn3-GFP (left panel) and MTLn3-GFP-ErbB1 cells (right panel) injected mice. d Lung metastases plotted against tumor weight of MTLn3-GFP-ErbB1 tumors bearing mice $(n=17)$

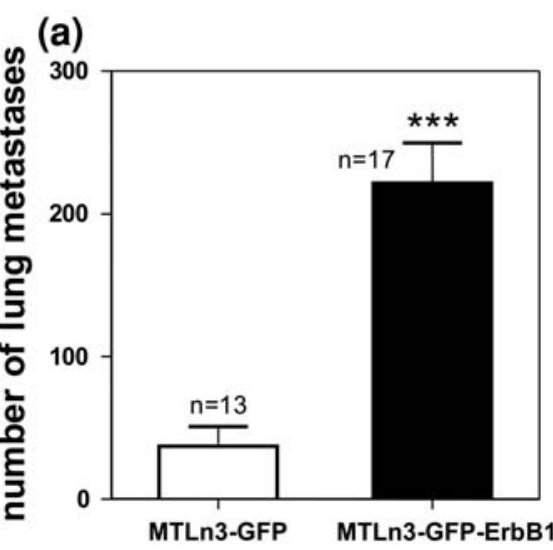

(c)

(b)

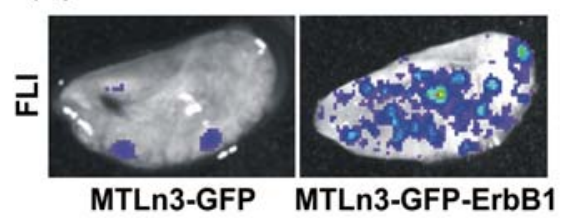

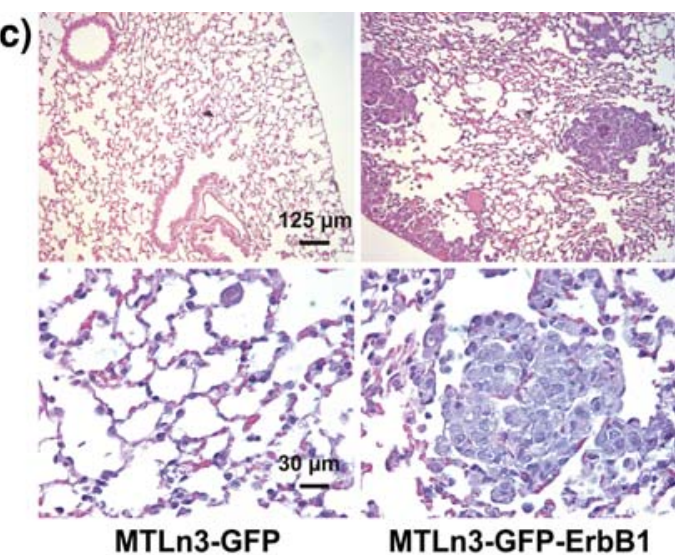

(d)

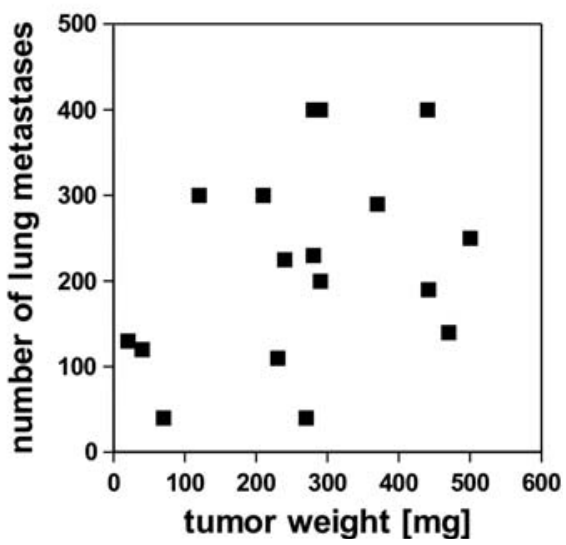

detect the tumor cells in blood. To determine the effects of increased ErbB1 expression on tumor growth and metastasis, MTLn3-GFP-ErbB1 and the control cell-line MTLn3-GFP were injected into the mammary fat pads of $\operatorname{Rag} 2^{-1-} \gamma \mathrm{c}^{-1-}$ mice, and tumor growth was monitored. All animals implanted with tumor cells developed tumors at the site of injection. The tumor growth of MTLn3-GFP cells was faster than that of MTln3-GFP-ErbB1 cells (Fig. 1c). Animals were sacrificed when the primary tumor reached similar volume for the two cell-lines between day 25 and 30. Indeed, there was no significant difference in tumor weight at the time of sacrifice (Fig. 1d). The animals were checked for spontaneous metastasis efficiency. Mice implanted with MTLn3-GFP-ErbB1 cells had significantly more lung metastases than mice implanted with MTLn3GFP cells (Fig. 2a) also evidence by fluorescence imaging (Fig. 2b). Histological analysis of the lungs showed an absence of micro-metastases in the MTLn3-GFP group (Fig. 2c). In the lungs of mice implanted with MTLn3GFP-ErbB1 cells, many macro-metastases could be detected on the surface and also throughout the lungs (Fig. 2c). No correlation was founded between tumor weight and number of lung metastases (Fig. 2d). We considered whether the additional period allowed for mice with delayed tumor growth could account for the increased number of lung metastases in the MTLn3-GFP-ErbB1 group. Therefore, we performed an experiment where both groups were sacrificed after 25 days. The primary tumors of the MTLn3-GFP-ErbB1 group again showed reduced growth rate (supplementary data Fig. 2a) and tumor weight was much lower than the MTLn3-GFP tumors (supplementary data Fig. 2b). Despite this reduced tumor growth, there was a trend toward a greater number of lung metastases in ErbB1-expressing tumor bearing mice (supplementary data Fig. 2c). We conclude from these results that increased expression of ErbB1 in MTLn3 cells results in reduced tumor growth but increased number of lung metastasis in Rag mice.

ErbB1-driven metastasis in the $\operatorname{Rag} 2^{-1-} \gamma \mathrm{c}^{-/-}$mice is dependent on enhanced intravasation in the primary tumor

Intravasation is a crucial step in the process of metastasis formation and ErbB1 signaling is thought to be a key factor in this process. Intravasation can be evaluated by determining the number of tumor cells present in blood collected from the right atrium of the heart. Animals bearing MTLn3-GFP tumors had very few tumor cells/ml of blood, whereas animals bearing MTLn3-GFP-ErbB1 tumors had an average of 170 tumor cells/ml of blood (Fig. 3a, b). In conclusion, increased ErbB1 expression in MTLn3 cells 
(a)

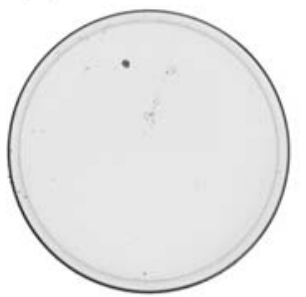

MTLn3-GFP

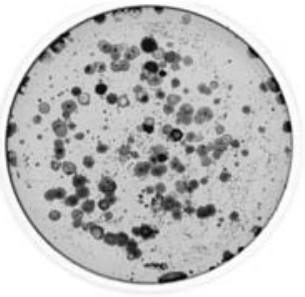

MTLn3-GFP-ErbB1 (b)

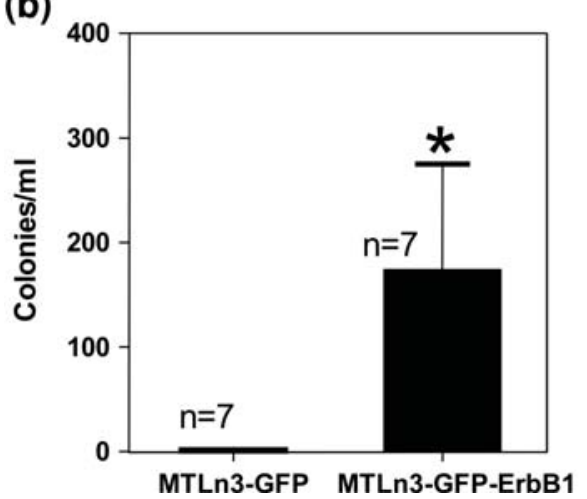

Fig. 3 ErbB1-driven metastasis is due to enhanced intravasation ability and not due to enhanced growth of metastases in the lungs. a Scan with Typhoon imager of two dishes where approximately $0.7 \mathrm{ml}$ of blood directly drawn from the right ventricle was cultured for 7 days. GFP colonies of blood collected from MTLn3-GFP inoculated mouse (left side) and from MTLn3-GFP-ErbB1 inoculated mouse (right side). b Tumor blood burden at the end point of the spontaneous metastasis assay. Colonies were counted after 7 days of culture

enhances intravasation and thereby metastasis formation in the $\operatorname{Rag} 2^{-1-} \gamma \mathrm{c}^{-/-}$mice.

Finally, we determined whether ErbB1-mediated enhanced lung metastasis was also related to increased homing, extravasation, or growth of the tumor cells in the lungs. For this purpose we compared the MTLn3GFP and MTLn3-GFP-ErbB1 cell lung metastasis following injected into the lateral tail vein (200,000 cells) of 5- to 7-week-old female Rag mice. Four weeks after injection, mice were sacrificed, and the lungs were removed and examined for metastases. While in the spontaneous metastasis assay significantly more metastases were observed in the MTLn3-GFP-ErbB1 transplanted group (Figs. 1, 2), animals receiving MTLn3GFP or MTLn3-GFP-ErbB1 cells in the tail vein developed a comparable number of metastatic lesions in the lungs (Fig. 3c, d). These results indicate that the enhanced ErbB1-dependent metastasis formation from the primary tumor is principally determined by intravasation and/or enhanced invasion rather than homing events at the target organ.
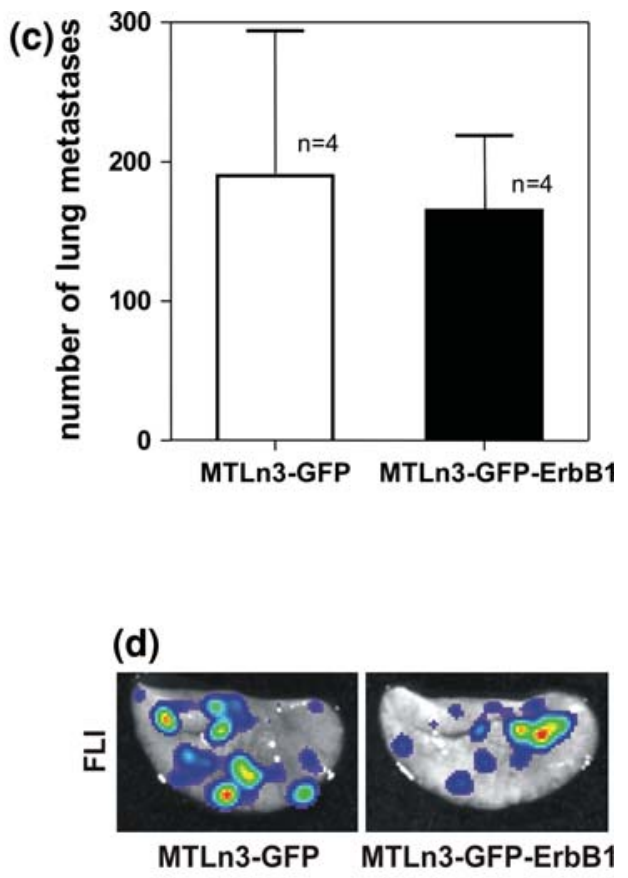

(MTLn3-GFP versus MTLn3-GFP-ErbB1, $P<0.05$ ). c Lung metastases counted with a fluorescent microscope at $\times 10$ magnification (only one side of the left lung) at the end point (day 28) of the experimental metastasis assays (tail vein MTLn3-GFP versus tail vein MTLn3-GFP-ErbB1, $P>0.05)$. d Lung metastases visualized with FLI after experimental metastasis assay with MTLn3-GFP and MTLn3-GFP-ErbB1 cells only

NK cells do not interfere with primary tumor growth

Previously, we demonstrated a negative role of NK immune cells in lung metastasis formation in Fischer 344 rats [27]. We analyzed several immune deficient mouse models for their capacity to support orthotopic breast cancer metastasis formation. Since ErbB1 enhances intravasation of MTLn3 tumor cells and GFP enables FLI and easy counting of individual lung metastases [14, 30, 31], we used the MTLn3-GFP-ErbB1 cell line to screen for the most suitable orthotopic breast/tumor metastasis model. We selected four mouse strains with different immunodeficiency backgrounds (see Table 1). The conventional nude mice still possess B lymphocytes and NK cells. Severe combined immunodeficiency (SCID) mice lack $\mathrm{T}$ and $\mathrm{B}$ cells but have NK cells. SCID Beige mice also lack NK cells $[6,18,20-23,25,32]$. $\operatorname{Rag}^{-1-} \gamma \mathrm{c}^{-/-}$mice lack both the adaptive and innate immune response. The common gamma $(\gamma \mathrm{c})$ knock-out mouse lacks functional receptors for many cytokines including IL-2, IL-4, IL-7, IL-9 and IL-15. As a consequence, lymphocyte development is greatly 
Table 1 Characteristics of immunodeficient mice used

\begin{tabular}{|c|c|c|c|}
\hline Strain & Adaptive immunity & Innate immunity & Reference \\
\hline BALB/c nu/nu 6 [Nude] & $\mathrm{T}$ cell deficiency & & {$[52]$} \\
\hline CB17/lcr-Prkdc ${ }^{\mathrm{SCID}} / \mathrm{Crl}[\mathrm{SCID}]$ & $\mathrm{T}$ and $\mathrm{B}$ cell deficiency & & {$[53-55]$} \\
\hline CB17/lcr.Cg-Prkdc ${ }^{\text {SCID }}$ Lyst $^{\text {tg }} / \mathrm{Crl}$ [SCID Beige] & $\mathrm{T}$ and $\mathrm{B}$ cell deficiency & Reduced NK cell function & {$[35,36,56]$} \\
\hline $\operatorname{Rag} 2^{-1-} \gamma \mathrm{c}^{-1-}[\mathrm{Rag}]$ & $\mathrm{T}$ and $\mathrm{B}$ cell deficiency & NK cell deficiency & {$[6,32,57,58]$} \\
\hline
\end{tabular}

compromised and NK cells are not present. Elimination of the residual $\mathrm{T}$ and $\mathrm{B}$ cells in the $\gamma \mathrm{c}^{-1-}$ background is obtained by crossing these mice to the recombinase activating gene 2 (Rag2) deficient mice $[6,32]$. All the mice were injected with 500,000 MTLn3-GFP-ErbB1 cells in the right thoracic mammary fat pad. We followed the primary tumor growth for 4 weeks and sacrificed the mice when the tumors reached an average size of $10 \mathrm{~mm} \times 10 \mathrm{~mm}$. The primary tumor growth rate in all mice strains except for the SCID Beige mouse was similar. The xenografts in the SCID Beige appeared to show delayed growth but the difference with the other mouse strains was not significant. Furthermore, the average tumor weight was comparable with the other three mouse strains (Fig. 4a, b). Overall the primary tumor volume varied between 300 and $500 \mathrm{~mm}^{3}$ (Fig. 4a) and tumor weight between 300 and $500 \mathrm{mg}$ (Fig. 4b). Both volume and weight were not significantly different between the four mouse strains. No difference was noticed in tumor organization, as determined by $\mathrm{H} \& \mathrm{E}$ staining (data not shown). No necrotic regions were observed in any of the tumors of the different mice strains. Since there was no significant difference in tumor weight especially between the SCID and the SCID Beige, the results suggest that NK cells do not interfere with the tumor growth of the MTLn3-GFPErbB1 cells.
Efficient orthotopic lung metastasis formation in the Rag2 $2^{-1-} \gamma \mathrm{c}^{-1-}$ and SCID Beige mice

After orthotopic transplantation of MTLn3 cells into the mammary gland of the four different mouse strains, primary tumors formed in all mice. Next we checked for the number of lung metastases after sacrificing the mice. The presence of GFP in the MTLn3 cells allowed the quantification of the lung metastases and classification according to their size by fluorescence microscopy (Fig. 5b). While there was no significant difference in primary tumor growth in the four different mouse strains (Fig. 4), almost no lung metastases were observed in nude or SCID mice (Fig. 5a). Many lung metastases were observed in both Rag2 ${ }^{-/-} \gamma \mathrm{c}^{-/-}$and SCID Beige mice, although the latter showed significantly fewer metastases than the Rag $2^{-1-}$ $\gamma \mathrm{c}^{-1-}$ mice (Fig. 5a). Visualization of individual metastases by immunofluorescence microscopy demonstrated that in the SCID Beige mice the size of the metastases ranged from less than 0.1 to $0.3 \mathrm{~mm}^{2}$ while in the $\mathrm{Rag} 2^{-1-} \mathrm{\gamma c}^{-1-}$ mice they were all above $0.3 \mathrm{~mm}^{2}$ (Fig. $5 \mathrm{~b}$ ). When the lungs were injected with ink, it was not possible to observe metastases in the SCID Beige; they were only visible with HE staining. However metastases were clearly visible in the Rag2 $2^{-I-} \gamma \mathrm{c}^{-/-}$mice (Fig. 5c). Since NK cells are still present in both nude and SCID mice but are absent in both
Fig. 4 MTLn3-GFP-ErbB1 cells have a similar growth rate in Nude, SCID, SCID Beige and $\operatorname{Rag} 2^{-l-} \gamma \mathrm{c}^{-/-}$mice. a Tumor growth rate monitored in the Nude $(n=5)$, SCID $(n=5)$, SCID Beige $(n=5)$ and $\operatorname{Rag} 2^{-\prime-} \gamma \mathrm{c}^{-/-}(n=6)$ mice by measuring the volume over the time period of the experiment. b Primary tumor weight at the end point of the spontaneous metastasis assay $(P>0.05)$
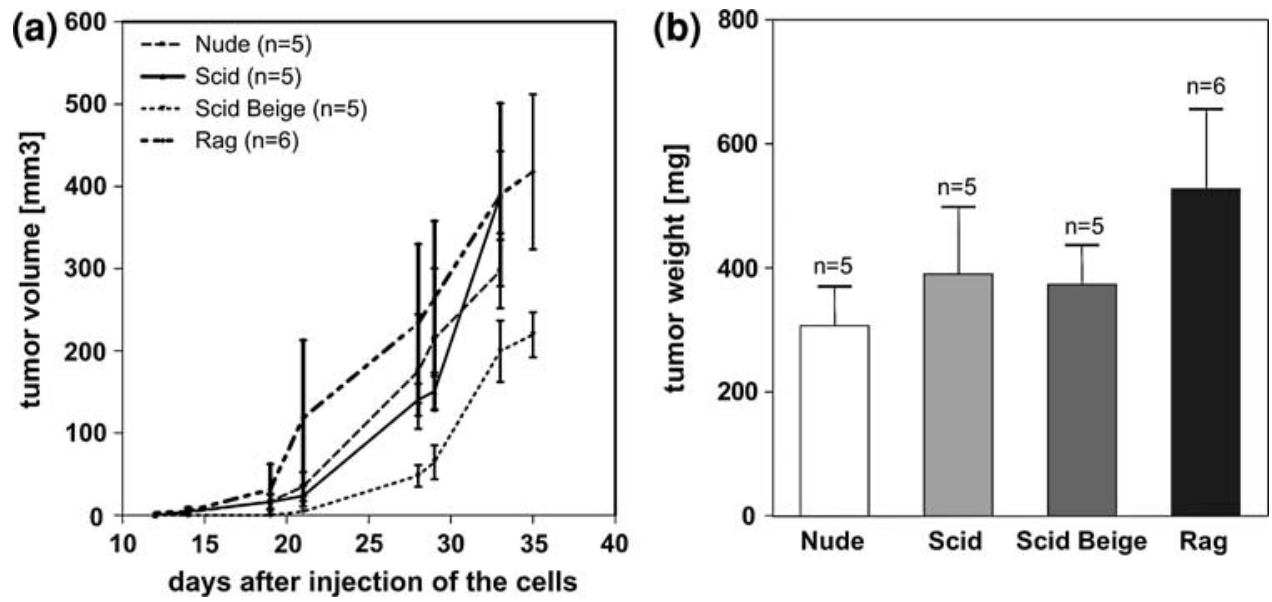
Fig. 5 NK cells interfere with lung metastasis formation. After sacrifice, the number of GFPpositive lung metastases was counted ex vivo using an inverted fluorescent microscope and a $\times 10$ objective lens. a Number of lung metastases at the end point of the spontaneous metastasis assay $(t$-test, $P<0.05)$. b Images of the four different sizes of lung metastases encountered during counting. Bar represents $100 \mu \mathrm{m}$. c After 5 weeks, lungs were isolated, injected with ink (right lobes) and lung (left lobe) sections were stained with $\mathrm{H} \& \mathrm{E}$ (a)

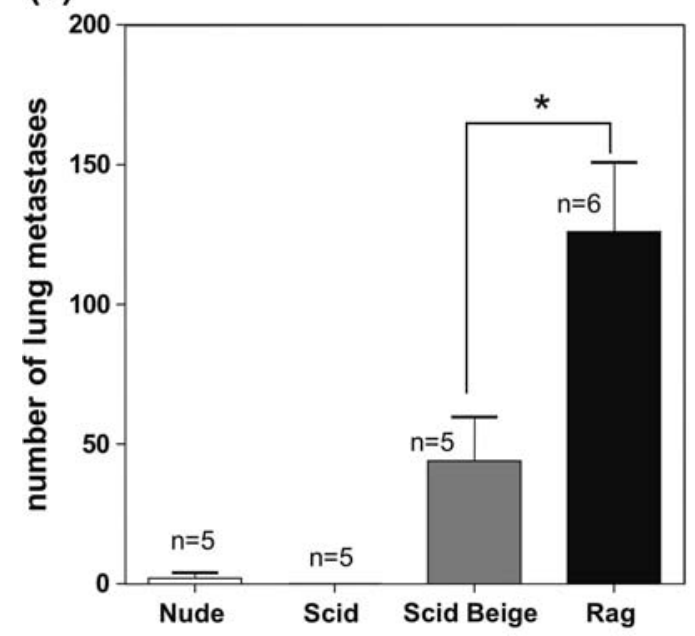

(b)

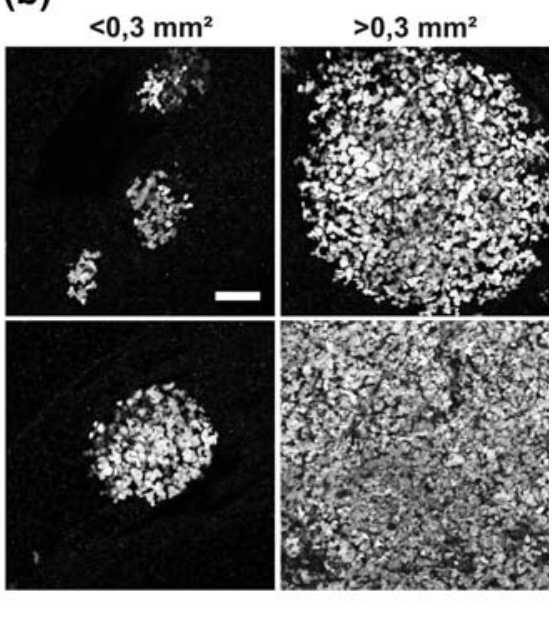

(c)

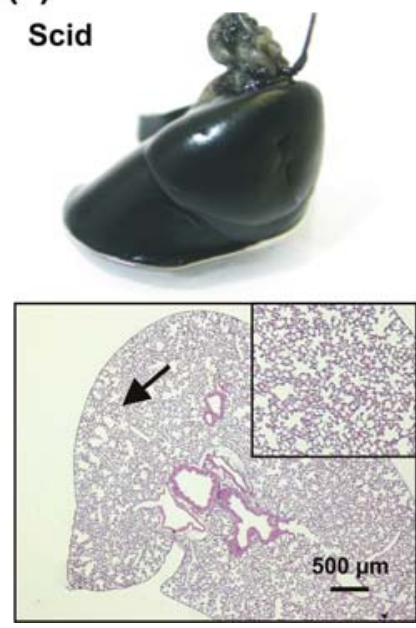

Scid Beige

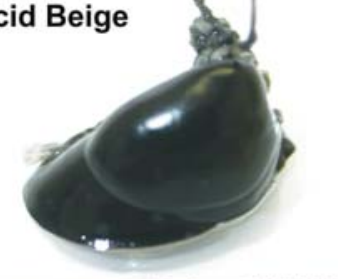

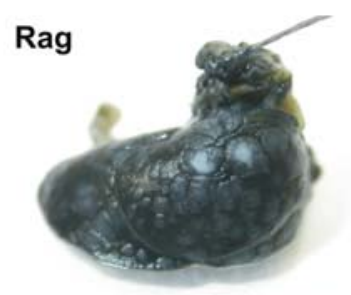
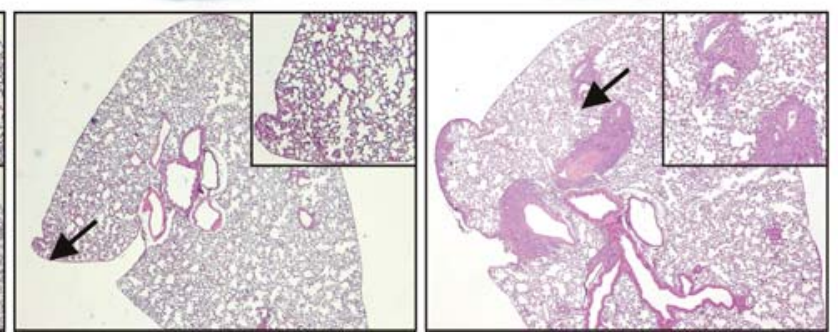

SCID Beige and Rag2 $2^{-1-} \gamma \mathrm{c}^{-/-}$mice, it shows that the presence of NK cells is the limiting factor for efficient lung metastasis formation in orthotopic breast cancer models. The beige mutation not only results in loss of cytotoxic $\mathrm{T}$ cells and selective impairment of NK cell functions but also in macrophage defects. Macrophages have been shown to influence MTLn3 lung metastasis formation [33, 34]. This could explain the lower average tumor weight and most probably the reduced number of lung metastases in the SCID Beige mice compared with the Rag2 $2^{-1-} \gamma^{-l-}$ mice $[35,36]$.

Efficient orthotopic lung metastasis formation in the Rag2 $2^{-/-} \gamma \mathrm{c}^{-/-}$using the 4T1-luc cell-line

Orthotopic transplantation of MTLn3-GFP-ErbB1 cells into the mammary gland of Rag2 $2^{-/-} \gamma \mathrm{c}^{-/-}$mice resulted in a high number of lung metastases. We also examined whether the $\mathrm{Rag}^{-/-} \gamma \mathrm{c}^{-/-}$mouse is a good recipient for another highly metastatic cell-line, 4T1-luc. This cell-line is supposed to metastasize easily to the lungs and other target organs in the syngeneic orthotopic mouse model the Balb/c mouse [37]. We injected 100,000 4T1-luc cells in the T4 mammary fat pad of both Rag2 $2^{-1-} \gamma \mathrm{c}^{-1-}$ and Balb/c mice. Over a time period of 3 weeks, we observed a significantly higher tumor growth rate in the Rag2 $2^{-1-} \gamma \mathrm{c}^{-/-}$mice (Fig. 6a), which we attribute to the influence of the immune system of the Balb/c mouse. At time of sacrifice, the primary tumor weight was similar in both mouse strains (Fig. 6b), however there were significantly more and larger lung metastases in the Rag $2^{-/-}$ $\gamma \mathrm{c}^{-1-}$ mice than in the Balb/c mice (Fig. 6c, d). After 3 weeks, only a high number of lung metastases formed by 4T1-luc cells could be observed in the Rag $2^{-1-} \gamma \mathrm{c}^{-1-}$ mouse model. We therefore conclude that $\operatorname{Rag} 2^{-l-} \gamma \mathrm{c}^{-/-}$mice represent a suitable breast cancer mouse model.

\section{Discussion}

Modeling metastasis in vivo is challenging but necessary for studying the mechanisms underlying the tumor cell 
Fig. 6 4T1-luc cells metastasize more efficiently in the $\operatorname{Rag} 2^{-1-} \gamma \mathrm{c}^{-1-}$ mice than in their syngeneic mouse model the Balb/c. a Tumor growth rate of the 4T1 cells monitored in the Rag2 $2^{-1-} \gamma \mathrm{c}^{-/-}$mice $(n=5)$ and in the Balb/C mice $(n=5)$ over 3 weeks. b Primary tumor weight at the end point of the spontaneous metastasis assay $(P>0.05)$. c Example of lung injected with ink (right lobe) to show number and size of metastases in both mouse models. d Lung metastases at the end point of the spontaneous metastasis assay ( $t$-test, $P=0.0040)$

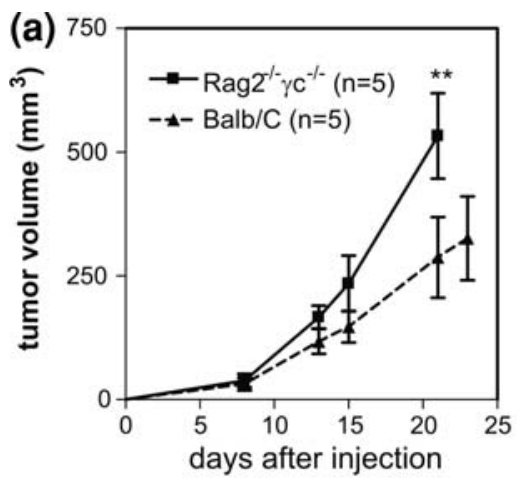

(c)
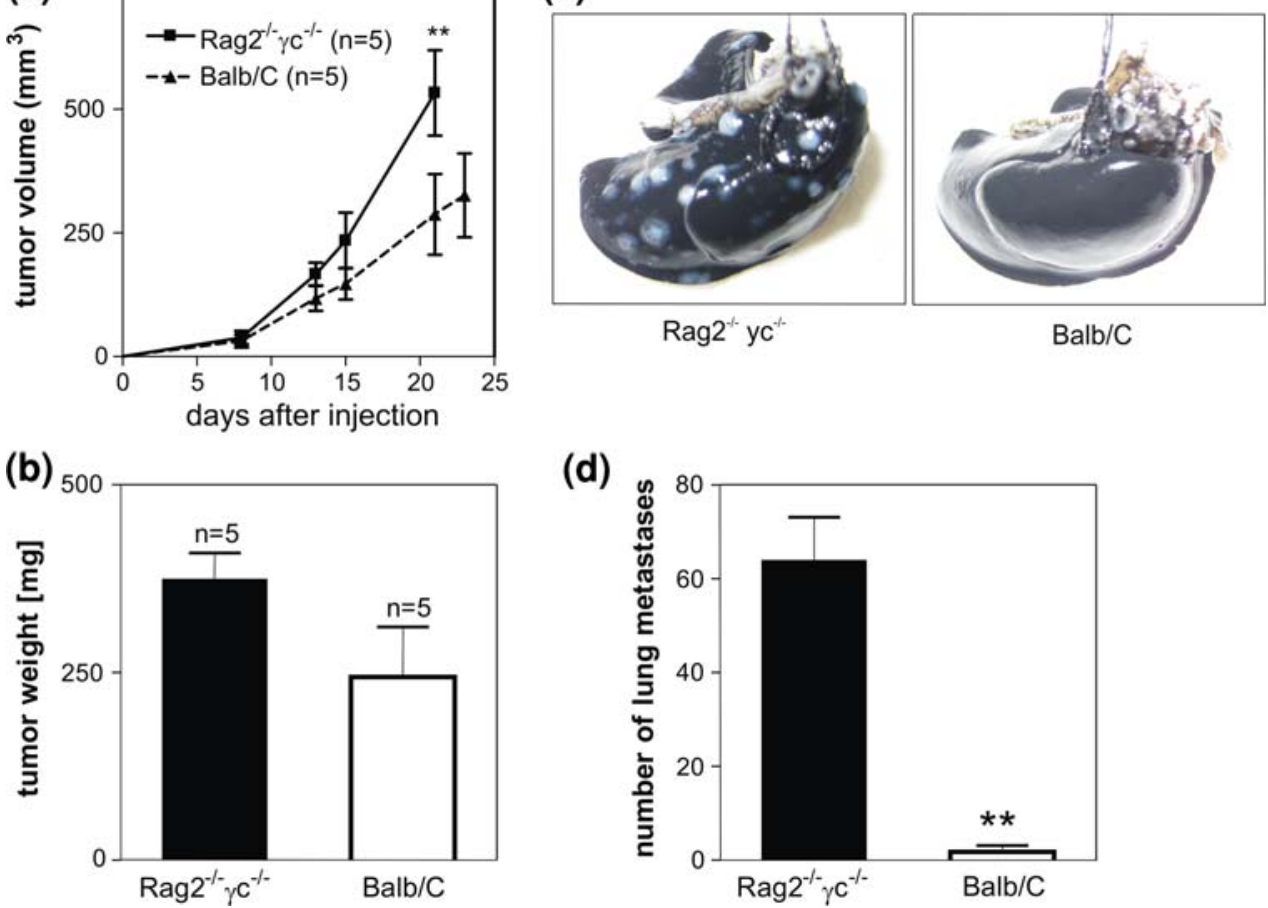

(d)

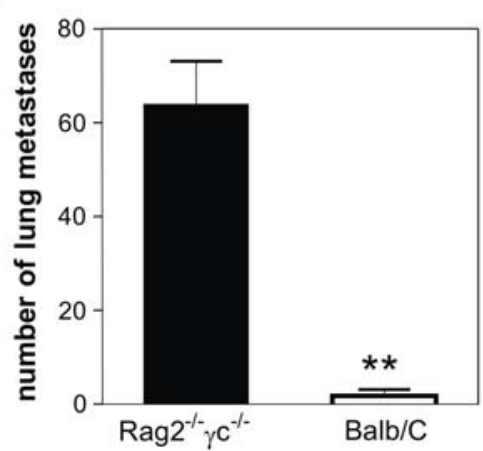

biological processes (e.g. cell migration) that enable certain cells to spread to other parts of the body [7]. EGFR-signaling is a pathway that is known to be involved in breast tumor progression. We examined whether EGFR overexpression in MTLn3 cells results in increased number of lung metastases in the Rag2 $2^{-/-} \gamma \mathrm{c}^{-/-}$mice. In contrast of previous findings $[12,13,16]$, our data indicate that increased expression of ErbB1 in the MTLn3 mammary adenocarcinoma cells results in slower primary tumor growth. In vitro, MTLn3-GFP-ErbB1 cells show an increased proliferation rate compared with control cells, suggesting instead that there may be a difference in cell survival. Indeed, MTLn3-GFP-ErbB1 cells are less able to tolerate culture at high density compared to MTLn3-GFP control cells. MTLn3-GFP-ErbB1 cells may be less tolerant of the fat pad injection procedure resulting in fewer surviving cells that can develop into a primary tumor.

ErbB1 overexpression enhances the ability to intravasate and, thus, provide sufficient seeding capacity to allow lung metastasis formation in the $\mathrm{Rag} 2^{-1-} \gamma \mathrm{c}^{-/-}$mice. Indeed, there were an increased number of tumor cells in the circulation of mice with ErbB1-expressing tumors, while few cells could be detected in the blood collected from control tumor bearing mice. Furthermore, the efficiency of lung colonization by MTLn3 cells (experimental metastasis assay) was independent of ErbB1 overexpression. These results are consistent with previous in vivo studies showing that ErbB1 expression can enhance invasiveness-most probably through increased chemotaxis to gradients of EGF $[33,34]$. The angiogenesis marker, CD31, failed to reveal differences in blood vessel density through the primary tumor of both groups (data not shown). The presence of macrophages in the $\mathrm{Rag} 2^{-1-} \gamma \mathrm{c}^{-/-}$mice allows the paracrine loop with tumor cells to take place and thus enhances invasiveness in response to EGF.

In this study, we have shown that the presence of remaining innate immune cells, including NK cells, in the nude and SCID mouse does not affect the growth of the primary tumor but inhibits the formation of lung metastases $[6,17,27,38]$. Indeed, we have found that the $\operatorname{Rag} 2^{-/-}$ $\mathrm{\gamma c}^{-/-}$mouse which lacks NK cells is an excellent recipient animal model to study breast tumor formation and progression when using MTLn3 overexpressing ErbB1 cells or 4T1 cells. Previous reports demonstrated the contribution of NK cells in tumor growth and metastasis [27, 39-42]. In particular, Dewan et al. [43] demonstrated the direct role of $\mathrm{NK}$ cells in tumor growth and metastasis using NOD/ $\mathrm{SCID} / \gamma \mathrm{c}^{\text {null }}$ (NOG) mice lacking T, B and NK cells which are similar to the $\mathrm{Rag}^{---} \gamma \mathrm{c}^{-l-}$ mice used in our study. They showed both increased efficiency of engraftment (subcutaneous inoculation only) and spontaneous metastasis of the human breast cancer cell-line MB-MDA-231 in the NOG mice compared to the SCID mice demonstrating the critical role of NK cells in tumor growth and metastasis [44]. In our study, we used the rat mammary adenocarcinoma MTLn3 cell-line overexpressing ErbB1. Although we did not observe a significant role for NK cells in engraftment and primary tumor growth, they did appear to be important for the formation of spontaneous lung metastases. Since the Rag2 $2^{-1-} \gamma \mathrm{c}^{-/-}$and NOG mice have 
similar immunodeficiencies, i.e. T, B, and NK cell reductions, similar results for the NOG and $\operatorname{Rag} 2^{-1-} \gamma \mathrm{c}^{-1-}$ mouse were to be expected. Zhang et al. [45] also conclude that $\mathrm{Rag} 2^{-I-} \gamma \mathrm{c}^{-/-}$mice, similar to $\mathrm{NOD} / \mathrm{SCID} / \gamma \mathrm{c}^{\text {null }}$ (NOG) mice, provide a suitable immunodeficient setting for human engraftment. Nevertheless, our results concerning efficient engraftment of the tumor cells do not show that NK cells play a role in this tumor progression step. Tumor growth in the SCID Beige mice was slightly delayed although these also lack NK cells. In fact, the beige mutation not only results in loss of cytotoxic $\mathrm{T}$ cells and selective impairment of NK cell functions but also in macrophage defects which could explain the lower average tumor weight and most probably the reduced number of lung metastases $[35,36]$. Indeed, it has been shown that macrophages can form a paracrine loop with tumor cells to enhance invasiveness in response to EGF stimulation [33, 34, 46, 47]. Reduced macrophage activity in SCID Beige mice could therefore explain why tumor growth and the number of lung metastases were reduced compared to Rag mice.

To further confirm that the Rag mouse is a suitable animal model for studying breast cancer progression, we injected two human xenografts models MDA-MB-231-luc [48] and a bone metastastic variant BO2-MDA-MB-231luc [49] cell-lines. These MDA-MB-231 cell lines poorly grew after orthotopic transplantation in Rag mice (data not shown). Typically, these cell lines are used in intracardial injection studies for bone metastasis model. The parental MB-MDA-231 cells do grow orthopically to $1 \mathrm{~cm}$ in diameter typically in 30-35 days after inoculation of $1 \times 10^{6}$ cells in SCID mice. After removal of the mammary fat pad tumors, mice are then sacrificed $4-5$ weeks later and few spontaneous metastases can be detected [50]. Although not tested, we anticipate that the parental MBMDA-231 cells would form orthotopic breast tumors in $\operatorname{Rag}^{-/-} \gamma \mathrm{c}^{-/-}$mice, but the necessary removal of the primary tumor for further metastases development as well as the ultimate number of lung metastasis formed, does not fit our requirements for an easy and reliable breast cancer/ metastasis animal model. In addition to our MTLn3 cell line, we also tested the 4T1 mouse mammary tumor cellline, which is known to be one of the only few breast cancer models with the capacity to metastasize efficiently to sites affected in human breast cancer [51]. When introduced orthotopically in the syngeneic Balb/c mouse model which possesses an intact immune system, 4T1 should be capable of metastasising to several organs typically affected in breast cancer $[12,13]$. However, when we injected the 4T1-luc cells in the Rag2 $2^{-1-} \gamma^{-/-}$and Balb/c mice, we could only detect lung metastases in the Rag2 $2^{-/-}$ $\gamma \mathrm{c}^{-l-}$ mice although a primary tumor formed in both mouse strains. Even after 3 weeks of experiment the primary tumor started to shrink in the Balb/c mice which has been described as a biphasic growth related to immune system function [37]. Indeed, Tao et al. observed a regression in weeks 2 through 4 in normal BALB/c mice which was associated with necrosis and infiltration of leukocytes. Biphasic tumor growth did not occur in athymic nude or SCID BALB/c mice suggesting involvement of an acquired immune response in the effect. We also injected the 4T1-luc in Balb/c mice purchased from different suppliers (data not shown). The results were similar: very few lung metastases detectable. In conclusion, our results suggest that NK cells play an important role in inhibiting metastasis formation but not in tumor growth.

In summary, ErbB1 overexpression in the MTln 3 cells delayed the primary tumor growth but was essential for efficient intravasation and lung metastasis formation. This suggests a role for ErbB1 expression in tumor cells to drive the biological processes that are essential in intravasation, such as cell motility. Secondly, we provide evidence for the crucial role of NK cells in metastasis formation but not in tumor growth. Metastasis formation is a key determinant of poor prognosis for breast cancer patients; enhancing NK activity is therefore a potential immunotherapeutic strategy for combatting this detrimental step.

The Rag2 $2^{-l-} \gamma \mathrm{c}^{-1-}$ mouse model, in combination with MTLn3-ErbB1 and 4T1 tumor cells, is a clinically relevant model for the study of breast cancer cell growth and metastasis. The 4T1 syngeneic metastatic breast cancer model may be useful to validate potential gene targets and processes implicated in cell migration and intravasation.

Acknowledgments We thank Jeffrey E. Segall for the GFPMTLn3-ErbB1 cells, valuable discussion and critically reading of the manuscript. We are also grateful to Leo Price for critically reading the manuscript. This work was financially supported by grants from the Dutch Cancer Society (UL 2006-3538 and UL 2007-3860), the EU FP7 Health Program Metafight (Grant agreement no.201862), the Netherlands Organization for Scientific Research (902-21-229 and 911-02-022) and TI Pharma (T3-107).

Open Access This article is distributed under the terms of the Creative Commons Attribution Noncommercial License which permits any noncommercial use, distribution, and reproduction in any medium, provided the original author(s) and source are credited.

\section{References}

1. Ferlay J, Autier P, Boniol M et al (2007) Estimates of the cancer incidence and mortality in Europe in 2006. Ann Oncol 18:581592. doi:10.1093/annonc/mdl498

2. Pantel K, Brakenhoff RH (2004) Dissecting the metastatic cascade. Nat Rev Cancer 4:448-456. doi:10.1038/nrc1370

3. Gupta GP, Massague J (2006) Cancer metastasis: building a framework. Cell 127:679-695. doi:10.1016/j.cell.2006.11.001 
4. van Nimwegen MJ, van de Water B (2007) Focal adhesion kinase: a potential target in cancer therapy. Biochem Pharmacol 73:597-609. doi:10.1016/j.bcp.2006.08.011

5. Vargo-Gogola T, Rosen JM (2007) Modelling breast cancer: one size does not fit all. Nat Rev Cancer 7:659-672. doi:10.1038/ nrc2193

6. Cao X, Shores EW, Hu-Li J et al (1995) Defective lymphoid development in mice lacking expression of the common cytokine receptor gamma chain. Immunity 2:223-238. doi:10.1016/10747613(95)90047-0

7. Khanna C, Hunter K (2005) Modeling metastasis in vivo. Carcinogenesis 26:513-523. doi:10.1093/carcin/bgh261

8. Sharpless NE, Depinho RA (2006) The mighty mouse: genetically engineered mouse models in cancer drug development. Nat Rev Drug Discov 5:741-754. doi:10.1038/nrd2110

9. Ottewell PD, Coleman RE, Holen I (2006) From genetic abnormality to metastases: murine models of breast cancer and their use in the development of anticancer therapies. Breast Cancer Res Treat 96:101-113. doi:10.1007/s10549-005-9067-x

10. Neri A, Welch D, Kawaguchi T et al (1982) Development and biologic properties of malignant cell sublines and clones of a spontaneously metastasizing rat mammary adenocarcinoma. J Natl Cancer Inst 68:507-517

11. Marxfeld H, Staedtler F, Harleman JH (2006) Characterisation of two rat mammary tumour models for breast cancer research by gene expression profiling. Exp Toxicol Pathol 58:133-143. doi:10.1016/j.etp.2006.05.003

12. Hynes NE, Lane HA (2005) ERBB receptors and cancer: the complexity of targeted inhibitors. Nat Rev Cancer 5:341-354. doi:10.1038/nrc1609

13. Giancotti V (2006) Breast cancer markers. Cancer Lett 243:145159. doi:10.1016/j.canlet.2006.01.035

14. Condeelis J, Segall JE (2003) Intravital imaging of cell movement in tumours. Nat Rev Cancer 3:921-930. doi:10.1038/ nrc1231

15. Condeelis J, Singer RH, Segall JE (2005) The great escape: when cancer cells hijack the genes for chemotaxis and motility. Annu Rev Cell Dev Biol 21:695-718. doi:10.1146/annurev. cellbio.21.122303.120306

16. Xue C, Wyckoff J, Liang F et al (2006) Epidermal growth factor receptor overexpression results in increased tumor cell motility in vivo coordinately with enhanced intravasation and metastasis. Cancer Res 66:192-197. doi:10.1158/0008-5472.CAN-05-1242

17. Cerwenka A, Lanier LL (2001) Natural killer cells, viruses and cancer. Nat Rev Immunol 1:41-49. doi:10.1038/35095564

18. Garofalo A, Chirivi RG, Scanziani E et al (1993) Comparative study on the metastatic behavior of human tumors in nude, beige/ nude/xid and severe combined immunodeficient mice. Invasion Metastasis 13:82-91

19. Sharkey FE, Fogh J (1979) Incidence and pathological features of spontaneous tumors in athymic nude mice. Cancer Res 39:833-839

20. Sebesteny A, Taylor-Papadimitriou J, Ceriani R et al (1979) Primary human breast carcinomas transplantable in the nude mouse. J Natl Cancer Inst 63:1331-1337

21. Rae-Venter B, Reid LM (1980) Growth of human breast carcinomas in nude mice and subsequent establishment in tissue culture. Cancer Res 40:95-100

22. Phillips RA, Jewett MA, Gallie BL (1989) Growth of human tumors in immune-deficient scid mice and nude mice. Curr Top Microbiol Immunol 152:259-263

23. Zietman AL, Sugiyama E, Ramsay JR et al (1991) A comparative study on the xenotransplantability of human solid tumors into mice with different genetic immune deficiencies. Int $\mathrm{J}$ Cancer 47:755-759. doi:10.1002/ijc.2910470522

24. Kubota T, Yamaguchi H, Watanabe M et al (1993) Growth of human tumor xenografts in nude mice and mice with severe combined immunodeficiency (SCID). Surg Today 23:375-377. doi:10.1007/BF00309059

25. Clarke R (1996) Human breast cancer cell line xenografts as models of breast cancer. The immunobiologies of recipient mice and the characteristics of several tumorigenic cell lines. Breast Cancer Res Treat 39:69-86. doi:10.1007/BF01806079

26. van Nimwegen MJ, Verkoeijen S, van Buren L et al (2005) Requirement for focal adhesion kinase in the early phase of mammary adenocarcinoma lung metastasis formation. Cancer Res 65:4698-4706. doi:10.1158/0008-5472.CAN-04-4126

27. van Nimwegen MJ, Verkoeijen S, Kuppen PJ et al (2007) An improved method to study NK-independent mechanisms of MTLn3 breast cancer lung metastasis. Clin Exp Metastasis 24(5): 379-387

28. Neri A, Nicolson GL (1981) Phenotypic drift of metastatic and cell-surface properties of mammary adenocarcinoma cell clones during growth in vitro. Int J Cancer 28:731-738. doi:10.1002/ ijc. 2910280612

29. Huigsloot M, Tijdens IB, Mulder GJ et al (2002) Differential regulation of doxorubicin-induced mitochondrial dysfunction and apoptosis by Bcl-2 in mammary adenocarcinoma (MTLn3) cells. J Biol Chem 277:35869-35879. doi:10.1074/jbc.M200378200

30. Condeelis JS, Wyckoff J, Segall JE (2000) Imaging of cancer invasion and metastasis using green fluorescent protein. Eur J Cancer 36:1671-1680

31. Wyckoff JB, Jones JG, Condeelis JS et al (2000) A critical step in metastasis: in vivo analysis of intravasation at the primary tumor. Cancer Res 60:2504-2511

32. Shinkai Y, Rathbun G, Lam KP et al (1992) RAG-2-deficient mice lack mature lymphocytes owing to inability to initiate V(D)J rearrangement. Cell 68:855-867. doi:10.1016/0092-8674(92) 90029-C

33. Wyckoff J, Wang W, Lin EY et al (2004) A paracrine loop between tumor cells and macrophages is required for tumor cell migration in mammary tumors. Cancer Res 64:7022-7029. doi:10.1158/0008-5472.CAN-04-1449

34. Wyckoff JB, Wang Y, Lin EY et al (2007) Direct visualization of macrophage-assisted tumor cell intravasation in mammary tumors. Cancer Res 67:2649-2656. doi:10.1158/00085472.CAN-06-1823

35. Roder J, Duwe A (1979) The beige mutation in the mouse selectively impairs natural killer cell function. Nature 278:451453. doi:10.1038/278451a0

36. Roder JC (1979) The beige mutation in the mouse I A stem cell predetermined impairment in natural killer cell function. J Immunol 123:2168-2173

37. Tao K, Fang M, Alroy J et al (2008) Imagable $4 \mathrm{~T} 1$ model for the study of late stage breast cancer. BMC Cancer 8:228. doi:10.1186/ 1471-2407-8-228

38. Wu J, Lanier LL (2003) Natural killer cells and cancer. Adv Cancer Res 90:127-156. doi:10.1016/S0065-230X(03)90004-2

39. Ben Eliyahu S, Page GG, Yirmiya R et al (1996) Acute alcohol intoxication suppresses natural killer cell activity and promotes tumor metastasis. Nat Med 2:457-460. doi:10.1038/nm0496-457

40. Ben Eliyahu S, Page GG, Yirmiya R et al (1999) Evidence that stress and surgical interventions promote tumor development by suppressing natural killer cell activity. Int J Cancer 80:880-888. doi:10.1002/(SICI) 1097-0215(19990315)80:6<880::AIDIJC14>3.0.CO;2-Y

41. Bouzahzah B, Albanese C, Ahmed F et al (2001) Rho family GTPases regulate mammary epithelium cell growth and metastasis through distinguishable pathways. Mol Med 7:816-830

42. Melamed R, Rosenne E, Shakhar K et al (2005) Marginating pulmonary-NK activity and resistance to experimental tumor metastasis: suppression by surgery and the prophylactic use of a beta-adrenergic antagonist and a prostaglandin synthesis 
inhibitor. Brain Behav Immun 19:114-126. doi:10.1016/ j.bbi.2004.07.004

43. Dewan MZ, Terunuma H, Ahmed S et al (2005) Natural killer cells in breast cancer cell growth and metastasis in SCID mice. Biomed Pharmacother 59(Suppl 2):S375-S379. doi:10.1016/ S0753-3322(05)80082-4

44. Beckhove P, Schutz F, Diel IJ et al (2003) Efficient engraftment of human primary breast cancer transplants in nonconditioned NOD/Scid mice. Int J Cancer 105:444-453. doi:10.1002/ ijc. 11125

45. Zhang B, Duan Z, Zhao Y (2008) Mouse models with human immunity and their application in biomedical research. J Cell Mol Med. doi:10.1111/j.1582-4934.2008.00347.x

46. Condeelis J, Pollard JW (2006) Macrophages: obligate partners for tumor cell migration, invasion, and metastasis. Cell 124:263266. doi:10.1016/j.cell.2006.01.007

47. Robinson-Smith TM, Isaacsohn I, Mercer CA et al (2007) Macrophages mediate inflammation-enhanced metastasis of ovarian tumors in mice. Cancer Res 67:5708-5716. doi:10.1158/00085472.CAN-06-4375

48. Peyruchaud O, Winding B, Pecheur I et al (2001) Early detection of bone metastases in a murine model using fluorescent human breast cancer cells: application to the use of the bisphosphonate zoledronic acid in the treatment of osteolytic lesions. J Bone Miner Res 16:2027-2034. doi:10.1359/jbmr.2001.16.11.2027

49. Wetterwald A, van der Pluijm G, Que I et al (2002) Optical imaging of cancer metastasis to bone marrow: a mouse model of minimal residual disease. Am J Pathol 160:1143-1153

50. Sossey-Alaoui K, Safina A, Li X et al (2007) Down-regulation of WAVE3, a metastasis promoter gene, inhibits invasion and metastasis of breast cancer cells. Am J Pathol 170:2112-2121. doi:10.2353/ajpath.2007.060975

51. Lelekakis M, Moseley JM, Martin TJ et al (1999) A novel orthotopic model of breast cancer metastasis to bone. Clin Exp Metastasis 17:163-170. doi:10.1023/A:1006689719505

52. Gershwin ME, Merchant B, Gelfand MC et al (1975) The natural history and immunopathology of outbred athymic (nude) mice. Clin Immunol Immunopathol 4:324-340. doi:10.1016/00901229(75)90002-1

53. McCune JM, Namikawa R, Kaneshima H et al (1988) The SCIDhu mouse: murine model for the analysis of human hematolymphoid differentiation and function. Science 241:1632-1639. doi:10.1126/science. 2971269

54. Dorshkind K, Pollack SB, Bosma MJ et al (1985) Natural killer (NK) cells are present in mice with severe combined immunodeficiency (scid). J Immunol 134:3798-3801

55. Schuler W, Bosma MJ (1989) Nature of the scid defect: a defective VDJ recombinase system. Curr Top Microbiol Immunol 152:55-62

56. Mosier DE, Stell KL, Gulizia RJ et al (1993) Homozygous scid/ scid; beige/beige mice have low levels of spontaneous or neonatal $\mathrm{T}$ cell-induced B cell generation. J Exp Med 177:191-194. doi:10.1084/jem.177.1.191

57. Colucci F, Soudais C, Rosmaraki E et al (1999) Dissecting NK cell development using a novel alymphoid mouse model: investigating the role of the c-abl proto-oncogene in murine $\mathrm{NK}$ cell differentiation. J Immunol 162:2761-2765

58. Greenberg PD, Riddell SR (1999) Deficient cellular immunityfinding and fixing the defects. Science 285:546-551. doi:10.1126/ science.285.5427.546 